\title{
Confocal laser scanning microscopy to estimate nanoparticles' human skin penetration in vitro
}

\author{
This article was published in the following Dove Press journal: \\ International Journal of Nanomedicine \\ 31 October 2017 \\ Number of times this article has been viewed
}

\author{
Ying Zou ${ }^{1,2, *}$ \\ Anna Celli ${ }^{2,3, *}$ \\ Hanjiang Zhu ${ }^{2, *}$ \\ Akram Elmahdy ${ }^{2}$ \\ Yachao $\mathrm{Cao}^{2}$ \\ Xiaoying $\mathrm{Hui}^{2}$ \\ Howard Maibach ${ }^{2}$ \\ 'Skin \& Cosmetic Research \\ Department, Shanghai Skin Disease \\ Hospital, Shanghai, People's \\ Republic of China; ${ }^{2}$ Department of \\ Dermatology, School of Medicine, \\ University of California San \\ Francisco, San Francisco, CA, USA; \\ ${ }^{3}$ San Francisco Veterans Medical \\ Center, San Francisco, CA, USA \\ *These authors contributed equally \\ to this work
}

Correspondence: Ying Zou

Skin \& Cosmetic Research Department,

Shanghai Skin Disease Hospital,

I 278 Baode Road, Shanghai 200443,

People's Republic of China

Tel +86 I39 I648 I856

Email zouyingsh@163.com
Objective: With rapid development of nanotechnology, there is increasing interest in nanoparticle (NP) application and its safety and efficacy on human skin. In this study, we utilized confocal laser scanning microscopy to estimate NP skin penetration.

Methods: Three different-sized polystyrene NPs marked with red fluorescence were applied to human skin, and Calcium Green 5N was used as a counterstain. Dimethyl sulfoxide (DMSO) and ethanol were used as alternative vehicles for NPs. Tape stripping was utilized as a barrierdamaged skin model. Skin biopsies dosed with NPs were incubated at $4^{\circ} \mathrm{C}$ or $37^{\circ} \mathrm{C}$ for 24 hours and imaged using confocal laser scanning microscopy.

Results: NPs were localized in the stratum corneum (SC) and hair follicles without penetrating the epidermis/dermis. Barrier alteration with tape stripping and change in incubation temperature did not induce deeper penetration. DMSO enhanced NP SC penetration but ethanol did not.

Conclusion: Except with DMSO vehicle, these hydrolyzed polystyrene NPs did not penetrate intact or barrier-damaged human "viable" epidermis. For further clinical relevance, in vivo human skin studies and more sensitive analytic chemical methodology are suggested.

Keywords: nanoparticles, skin penetration, stratum corneum, confocal laser scanning microscopy, tape stripping

\section{Background}

Nanotechnology, a rapidly emerging field, provides new techniques and tools. ${ }^{1}$ Nanomaterials including nanoparticles (NPs), nanoemulsions and nanosomes are widely used in pharmacology, cosmetics, medicines, etc. ${ }^{2}$ NPs, defined as particles at least one dimension smaller than $100 \mathrm{~nm}$, have been engineered for carrying drug payloads, imaging contrast agents, or gene therapeutics for diagnosing and treating diseases, and ingredients in cosmetics. ${ }^{3-5}$ With increasing NP applications, investigations focus on optimization in therapeutic/cosmetic use and their health hazards. Since skin is a major target tissue for the exposure of NPs, the assessment of NP skin penetration has attracted great attention. ${ }^{2}$

General pathways of skin absorption occur via appendages and through stratum corneum (SC) to underling layers. ${ }^{6}$ Skin conditions and NP properties, such as size, shape and charge, are crucial for skin permeability. ${ }^{7}$ Investigation of skin penetration versus different parameters should provide valuable knowledge on promotion or minimization of NP skin penetration.

Qualitative microscopy visualization techniques, including scanning electron microscopy (SEM), transmission electron microscopy (TEM), fluorescence microscopy and confocal and multiphoton laser scanning microscopy, offer opportunities of noninvasiveness, high sensitivity and high spatial resolution analysis of NP skin penetration.? 
Conventional microscopy such as light microscopy, SEM and TEM have limitation of artifacts due to sample staining and/or mechanical section, whereas confocal and multiphoton laser scanning microscopy enable researchers to obtain three-dimensional image of NP distribution at micrometer resolution by way of "optical sectioning." ${ }^{8}$ Occupied fluorescence confocal laser scanning microscopy can detect NP distribution in lifetime information.

Despite increasing studies on penetration and mechanism of NP distribution in skin, behavior of NPs remains sub judice with conflicting results reported. ${ }^{2,8}$ Factors affecting NP skin penetration, including physicochemical NP properties, formulation and environmental and skin conditions, make it difficult to draw general conclusions on NP skin penetration. ${ }^{8}$

In the present study, penetration pathway of fluorescencemarked NPs in ex vivo human skin samples was tracked utilizing spectral confocal microscopy; the impact of skin condition, incubation temperature, NP size and vehicles on NP distribution in skin were assessed visually.

\section{Materials and methods}

Calcium Green 5N (CG5N; Thermo Fisher Scientific, Waltham, MA, USA) was employed as skin staining for autofluorescence. Polystyrene NPs sized $25 \mathrm{~nm}, 50 \mathrm{~nm}$ and $100 \mathrm{~nm}$, namely red fluorescent polymer microspheres R25/R50 and R100 in water, were obtained from Thermo Fisher Scientific. Dimethyl sulfoxide (DMSO; Acros, Morris Plains, NJ, USA) and 99\% ethanol (Sigma-Aldrich Co., St Louis, MO, USA) were used as alternative vehicles for NP dissolution. DMSO and ethanol concentration in NP dispersion was $80 \%$.

Human skin was excised from five donors (age $42-55$ years) with no medical history of dermatological disease undergoing abdominal plastic surgery after their written informed consent was completed. The procedures were performed under protocols approved by the University of California, San Francisco, and in accordance with the principles expressed in the Declaration of Helsinki. This study was approved by the University of California Institutional Review Board. After excision, the subcutaneous fatty tissue was removed by surgical scalpel.

After cutting into rectangular pieces, each sample was incubated with a $50 \mu \mathrm{M}$ solution of CG5N with the dermal side in contact with dye solution. Samples were incubated overnight in the dark at $4^{\circ} \mathrm{C}$ to enable dye penetration. Samples were rinsed in PBS three times to remove excess dye and dried with paper tissue. After cutting into $2 \times 2 \mathrm{~cm}^{2}$ pieces, specimens were fixed flatly on diffusion cells (PermeGear Inc., Hellertown, PA, USA) with the dermal side in contact with media.

Red fluorescent polymer microspheres of varied size and/or dissolved in different vehicles were dosed on the surface of samples from the dosing hole on diffusion cell and then incubated at $4^{\circ} \mathrm{C}$ for 24 hours. After clearing excess NPs with PBS, specimens were mounted with SC in contact with a glass coverslip and secured on the stage for imaging. For each experiment, a sample without NP dosing was used as blank control. Imaging of NPs in DMSO and ethanol was compared with that of original NPs.

To observe the impact of incubation temperature, higher temperature $\left(37^{\circ} \mathrm{C}\right)$ was compared with routine one $\left(4^{\circ} \mathrm{C}\right)$, both incubated for 24 hours. For skin barrier-damaged studies, the specimens were tape stripped 20 times on the area of interest before securing on the perfusion chamber. The intact skin without tape stripping was used as control.

Imaging was performed using a Zeiss LSM 780 confocal microscopy system (Carl Zeiss Meditec AG, Jena, Germany). About $488 \mathrm{~nm}$ and $561 \mathrm{~nm}$ laser lines were used as excitation sources for CG5N and NP fluorescence, respectively. The emitted fluorescence was detected in two separate spectral channels centered at $550 \mathrm{~nm}$ and $637 \mathrm{~nm}$, respectively, for CG5N and NPs.

\section{Results}

\section{NPs of different size distributions in human skin (water vehicle)}

Figure 1 depicts the pattern of NP distribution in human skin. About 24 hours after topical application, red fluorescence of NPs was clearly observable and especially pronounced on the topmost skin layers. No penetration was observed in stained "viable" epidermal layers (green channel). A prominent NP signal was noticeable tracing hair follicles without spreading to the neighboring cells and tissues.

\section{Vehicles (DMSO and ethanol)}

Figure 2 shows NP distribution in human skin when $80 \%$ DMSO and $80 \%$ ethanol are used as vehicles. NPs dissolved in DMSO penetrated deeper; red fluorescence was detected in stratum granulosum layer but not in stratum spinosum. Imaging of specimens dosed with NPs in ethanol showed NPs deposited in SC layer without entering "viable" epidermis.

\section{Incubation temperature}

Figure 3 shows NP distribution in dosed specimens incubated at $4^{\circ} \mathrm{C}$ or $37^{\circ} \mathrm{C}$ for 24 hours. Higher temperature did not enhance the penetration depth of NPs. 

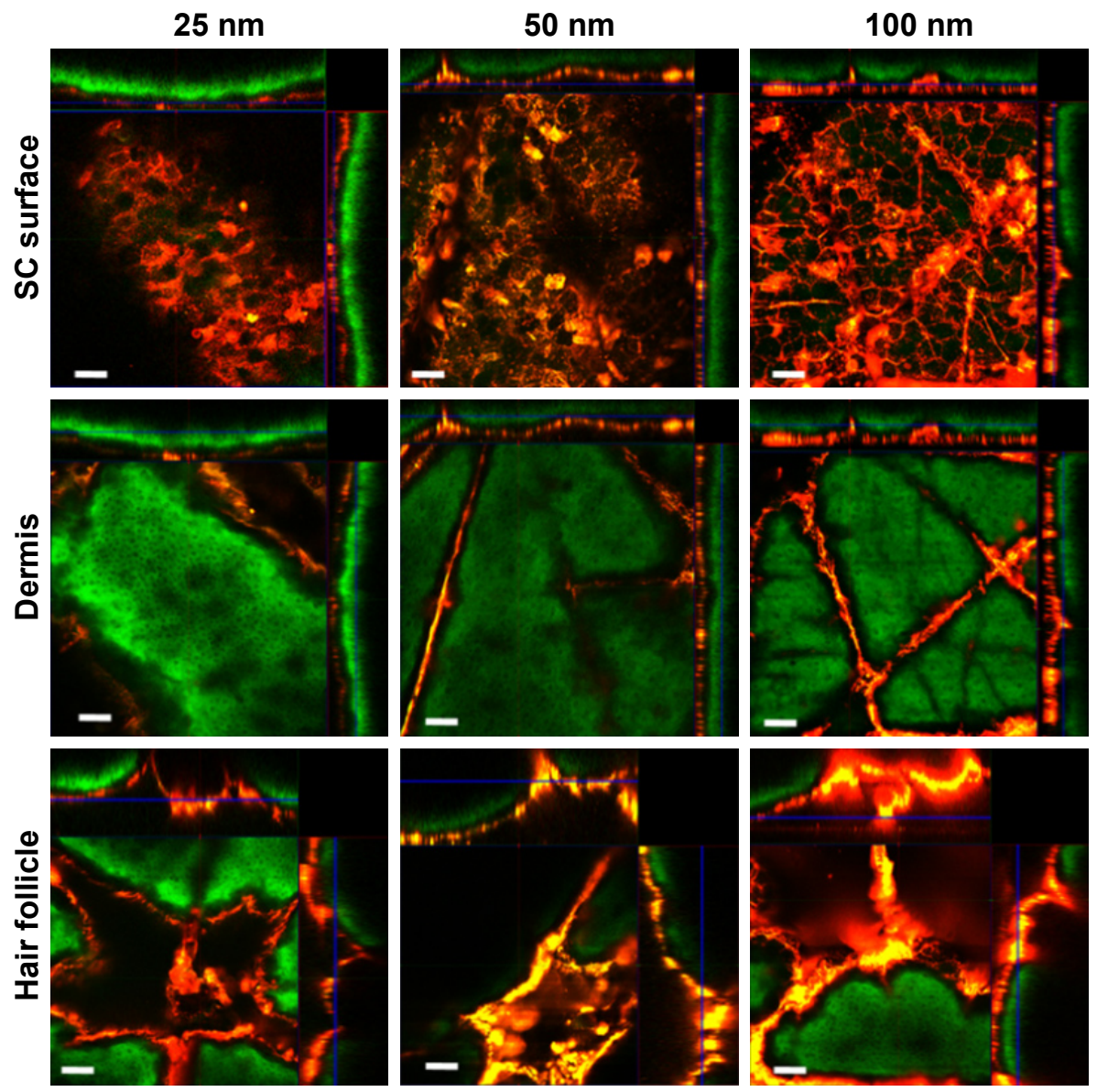

Figure I NPs of different size distributions in human skin.

Notes: Three different-sized NP distributions are shown. Red fluorescence shows the NPs and green fluorescence shows the skin samples. NPs (in red) distribute on the surface of SC and hair follicles. No penetration to deeper layer was observed. Scale bars $=50 \mu \mathrm{m}$.

Abbreviations: NPs, nanoparticles; SC, stratum corneum.

\section{NP distribution in barrier-damaged skin}

Figure 4 shows the image of skin penetration after tape stripping. Tape stripping permitted deeper SC penetration. The no-NP location gap between SC layer and granulosum layer became smaller in tape stripped skin than intact skin, but no traces of NPs presented in granulosum or deeper.

\section{Discussion}

NPs have wide applications in cosmetics, pharmaceutics and biomedicine. Penetration studies provided conflicting data. ${ }^{8}$ It is crucial to understand the skin permeability of NPs and its behavior in different skin layers. Clarification of factors that either hinder or enhance NP skin penetration may benefit the design of an "ideal" carrier or agent of NP for drugs and cosmetics. Moreover, since not only intentional applications of drugs and cosmetics were the routes for the exposure of NPs but also non-intentional ways and environmental exposure could introduce NPs on the skin, there is increasing focus on health risks of NPs. In spite of studies on skin penetration of NPs, the mechanism and relative factors remain unclear with some conflicting results reported. ${ }^{8}$

Several factors may affect skin penetration such as physicochemical properties of NPs, formulation (vehicle) and experimental factors. ${ }^{8}$

Physicochemical properties, such as size and surface charge of NPs, are key factors of NP skin penetration. ${ }^{9}$ Negatively charged NPs had a greater diffusion coefficient ${ }^{10}$ and penetrated skin more rapidly, ${ }^{11,12}$ while positive charges acted in an opposite way. Since there is negatively charged electrostatic interaction on skin surface, the potential incentive of charge effect could be a repulsive or attractive force between skin surface and negatively or positively charged NPs, respectively. ${ }^{13}$ This study focused on the effect of particle size on NP skin penetration; neutrally charged polystyrene NPs were chosen to eliminate influences of surface charge.

Converse results were reported on skin permeability of NPs with different sizes. ${ }^{14-17}$ Studies using animal skin rather 

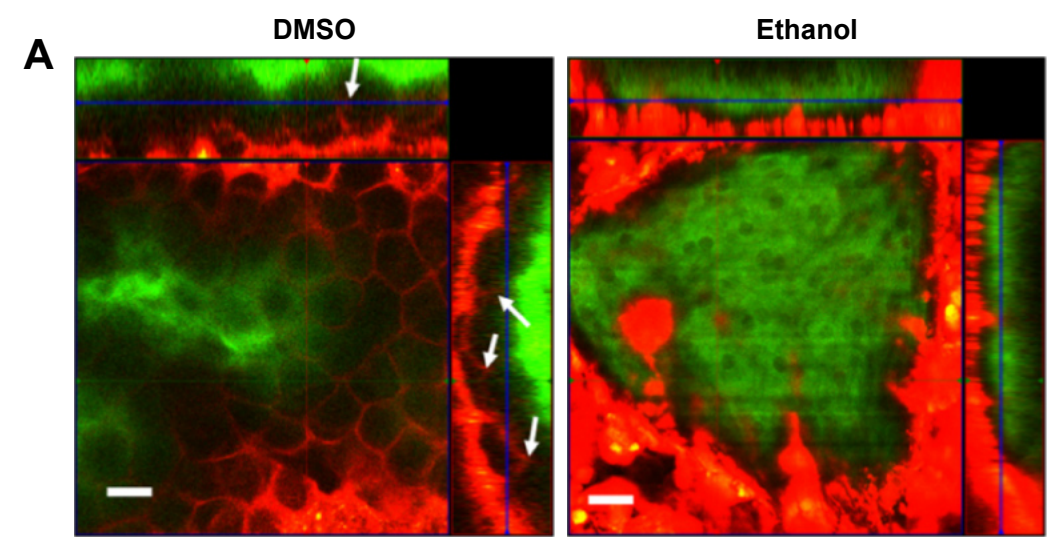

B
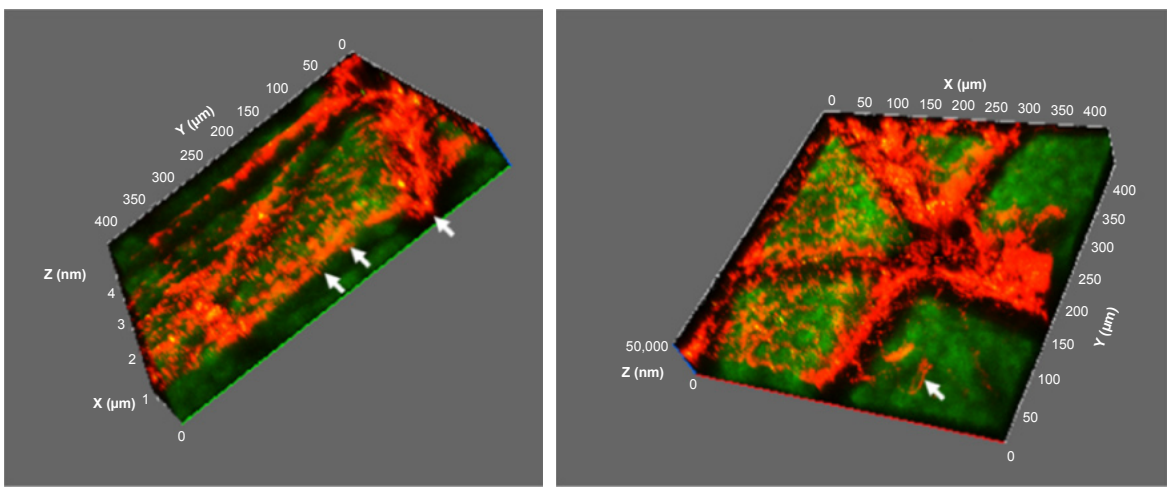

Figure 2 Distribution of NPs dissolved in DMSO and ethanol.

Notes: Red fluorescence of DMSO-dissolved NPs (size $25 \mathrm{~nm}$ ) is detected in stratum granulosum layer. Arrows show the penetration of NPs to deeper layer in longitudinal section (A, left) and 3D images (B). No red fluorescence of ethanol-dissolved NPs (size $25 \mathrm{~nm}$ ) was observed in granulosum and deeper layers $(\mathbf{A}$, right). Scale bars $=50 \mu \mathrm{m}$. Abbreviations: 3D, three-dimensional; DMSO, dimethyl sulfoxide; NPs, nanoparticles.
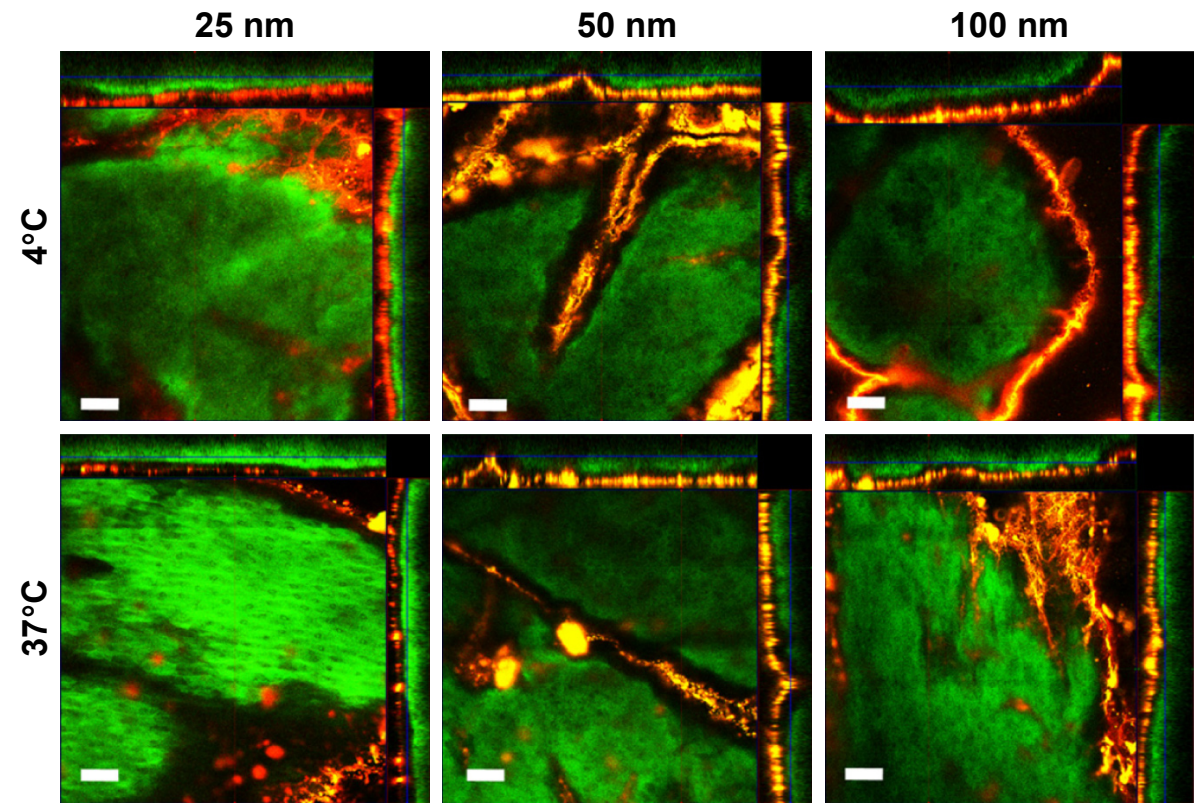

Figure 3 Distribution of NPs incubated in different temperatures.

Notes: Three different-sized NP distributions are shown. NPs (shown in red fluorescence) distribute in $\mathrm{SC}$ after incubation under both $4^{\circ} \mathrm{C}$ and $37^{\circ} \mathrm{C}$. No red fluorescence is shown in deeper layers. Scale bars $=50 \mu \mathrm{m}$.

Abbreviations: NPs, nanoparticles; SC, stratum corneum. 

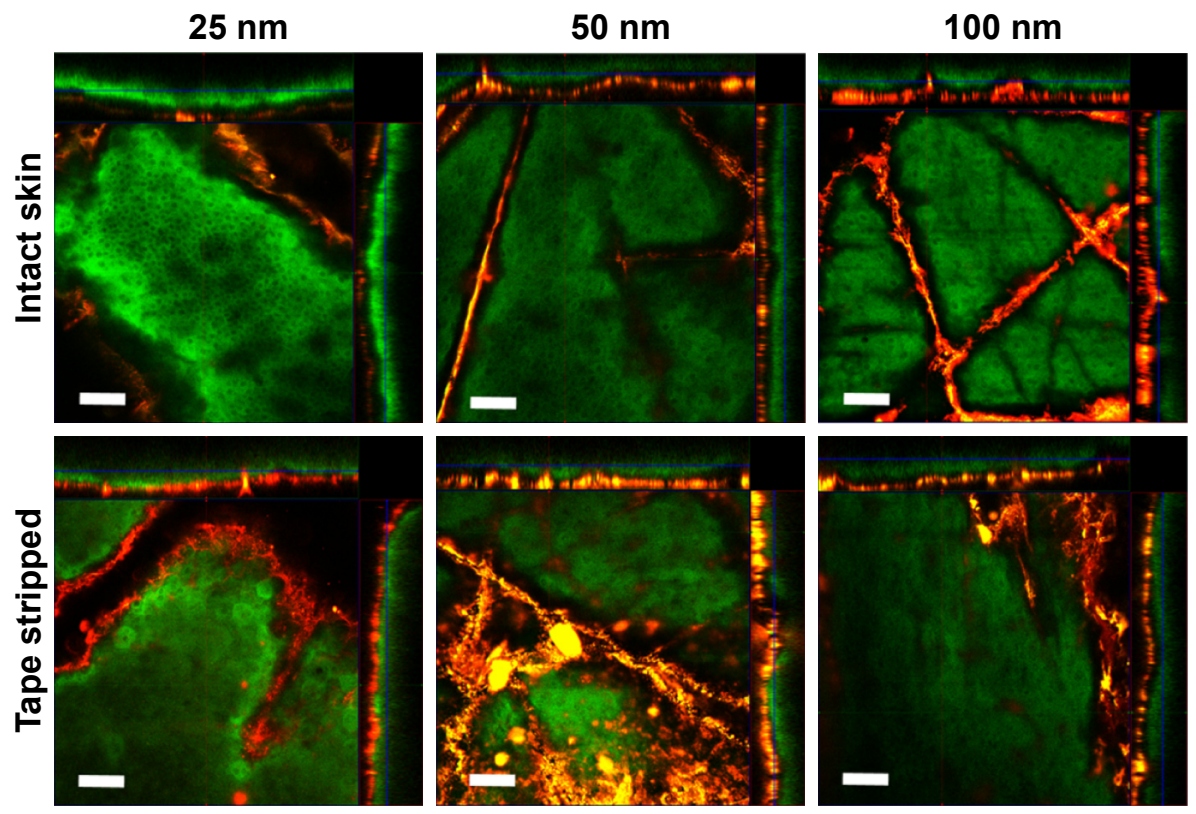

Figure 4 Distribution of NPs in barrier-damaged skin.

Notes: Three different-sized NP distributions in both intact and barrier-damaged skins are shown. The no-NP location gap between SC layer and granulosum layer became smaller in barrier-damaged skin than in intact skin, but no red fluorescence of NPs presented in granulosum and deeper layer. Scale bars $=50 \mu \mathrm{m}$.

Abbreviations: NPs, nanoparticles; SC, stratum corneum.

than human skin showed a positive result of skin penetration. ${ }^{8}$ Zvyagin et $\mathrm{al}^{18}$ suggested that animal skin represents a poor model for human skin for the studies on NP transdermal penetrability. Differences between these skins, especially hair follicle density, SC thickness, whole skin and skin lipid mass, ${ }^{19}$ could lead to different results. Due to structural and morphological differences between human and animal skin, excised human skin is regarded as a "gold standard" for in vitro skin penetration studies. ${ }^{20}$ Therefore, we employed excised human skin in this study. Three different-sized polystyrene NPs $(25 \mathrm{~nm}, 50 \mathrm{~nm}$ and $100 \mathrm{~nm}$ ) were assayed on their distributions in SC and hair follicles. Permeability of each sized NP made no difference in excised human skin, and no "viable" epidermal penetration was observed. The results were consistent with some reports using human skin ${ }^{8,18,21}$ and animal skin. ${ }^{16,17}$

Barrier function of SC against molecule penetration in general depends on its protein, lipid and water compartments. ${ }^{22}$ Vehicle may alter the nature of skin barrier or even the physical state of NPs, which makes it another factor affecting NP penetration. ${ }^{15}$ Labouta et $\mathrm{a}^{23}$ studied the effect of DMSO on the penetration of gold NPs (AuNPs, $\phi=10 \mathrm{~nm}$ ) in human skin and showed enhanced NP transport in the presence of DMSO. Kuo et $\mathrm{al}^{24}$ demonstrated ethanol as an enhancer of zinc oxide NPs $(\phi=10 \mathrm{~nm})$ in animal skin. Our study evaluated the effect of DMSO and ethanol on aqueous NP penetration. NPs dissolved in DMSO penetrated into deeper skin layers. However, ethanol did not alter NP penetration in the size range of 25-100 $\mathrm{nm}$ in human skin. Being absorbed into corneocytes, DMSO may change keratin conformation and then enhance NP penetration. ${ }^{23}$

Temperature is considered a critical factor in chemical penetration, ${ }^{25-27}$ however, no previous study investigated the influence of temperature on NP penetration to our best knowledge. In the current study, skin samples were incubated at $4^{\circ} \mathrm{C}$ and $37^{\circ} \mathrm{C}$ after dosage. No further penetration was detected at higher temperature, indicating that NP penetration was stable under different temperature conditions.

There are two general pathways for skin absorption: through SC and the underlying layers and along skin appendages. No NP penetration through SC was observed in this study, except when DMSO was used as an enhancer. Via hair follicles, NPs may reach deep into subcutaneous fat; however, hair follicle also contains an efficient barrier, which is similar to $\mathrm{SC}$ in upper part and features tight junction in lower part. This barrier inhibits NPs invading into viable cells. Thus, traces into hair follicles are not yet a deep absorption process, as they remain on the outside of the body by definition.

NPs stored in hair follicles could also be extruded by hair growth and sebum flow. Lademann et $\mathrm{al}^{28}$ found that NPs located in SC were removed after 24 hours, while that in hair follicles remained more than 10 days, whereas non-particles only stored in hair follicles up to 4 days. ${ }^{29}$ Therefore, hair 
follicles, representing an efficient reservoir for NPs, can be a potential target of NP carrier system for drug delivery, topical vaccination and allergenic potential. ${ }^{29,30}$

To investigate the permeability of NPs in barrier-damaged skin, we tape stripped samples mimicking barrier disruption. Tape stripping removed SC partially or totally, and it is a simple standard technique for the establishment of skin barrier-damaged model. ${ }^{30}$ However, NP penetration through tape stripped skin did not always lead to marked penetration enhancement. It varied qualitatively in magnitude from none $^{31-34}$ to some NPs detected. ${ }^{15,35,36}$ Differences may be affected by skin species, number of strips and type of tape used and/or NP characteristics. Our results noted that tape stripping (20 times) did not facilitate penetration of aqueous NPs (size 25-100 nm), thus NP skin penetration, even through barrier-impaired skin, is limited.

Limitations in this study include the following: 1) quantification method in visual measurement was not established; 2) reported data were applicable to experimental condition and may not be readily extracted to other NPs; and 3 ) verification in human in vivo would be a next step. To understand NP penetration of a barrier altering skin condition would be meaningful to NP application for diseased skin. More systemic studies are needed to clarify the biodistribution of NPs.

\section{Conclusion}

This study combined fluorescence of NPs and fluorescent counterstaining of epidermis to image NP transdermal pathway with different parameters, using confocal laser scanning microscopy. Hydrolyzed NPs in the size ranging from $25 \mathrm{~nm}$ to $100 \mathrm{~nm}$ confirmed their SC and hair follicle location. Barrier alteration with tape stripping and change in incubation temperature did not induce deeper penetration. DMSO enhanced NP penetration, but ethanol did not. There remain many important questions and technical challenges in nanotechnology, especially in dermatological science.

\section{Acknowledgments}

This research was supported by the Defense Threat Reduction Agency (DTRA) (grant: HDTRA1-14-0005 UCSF [BRBAA11-PerC-9-2-0054-Base]) and the Science Foundation of Shanghai Municipal Commission of Health and Family Planning (grant number 201440366). We wish to acknowledge the UCSF Laboratory for Cell Analysis for providing the equipment used in this investigation.

\section{Disclosure}

The authors report no conflicts of interest in this work.

\section{References}

1. Niemeyer CM. Nanoparticles, proteins, and nucleic acids: biotechnology meets materials science. Angew Chem Int Ed. 2001;40(22): 4128-4158.

2. DeLouise LA. Applications of nanotechnology in dermatology. J Invest Dermatol. 2012;132(3):964-975.

3. Moghimi SM, Hunter AC, Murray JC. Nanomedicine: current status and future prospects. FASEB J. 2005;19(3):311-330.

4. Nohynek GJ, Lademann J, Ribaud C, Roberts MS. Grey goo on the skin? Nanotechnology, cosmetic and sunscreen safety. Crit Rev Toxicol. 2007;37(3):251-277.

5. Huang HC, Barua S, Sharma G, Dey SK, Rege K. Inorganic nanoparticles for cancer imaging and therapy. J Control Release. 2011;155(3): 344-357.

6. Schneider M, Stracke F, Hansen S, Schaefer UF. Nanoparticles and their interactions with the dermal barrier. Dermatoendocrinol. 2009;1(4): 197-206.

7. Song Z, Anissimov YG, Zhao JB, et al. Background free imaging of upconversion nanoparticle distribution in human skin. J Biomed Opt. 2013;18(6):061215.

8. Labouta HI, Schneider M. Interaction of inorganic nanoparticles with the skin barrier: current status and critical review. Nanomedicine. 2013;9(1):39-54.

9. Sonavane G, Tomoda K, Sano A, Ohshima H, Terada H, Makino K. In vitro permeation of gold nanoparticles through rat skin and rat intestine: effect of particle size. Colloids Surf B Biointerfaces. 2008; 65(1):1-10.

10. Kim B, Han G, Toley BJ, Kim CK, Rotello VM, Forbes NS. Tuning payload delivery in tumour cylindroids using gold nanoparticles. Nat Nanotechnol. 2010;5(6):465-472.

11. Sinico C, Manconi M, Peppi M, Lai F, Valenti D, Fadda AM. Liposomes as carriers for denual delivery of tretinoin: in vitro evaluation of drug penneation and vesicle-skin interaction. J Control Release. 2005; 103(1):123-136.

12. Lee O, Jeong SH, Shin WU, Lee G, Oh C, Son SW. Influence of surface charge of gold nanorods on skin penetration. Skin Res Technol. 2013; 19(1):E390-E396.

13. Lee $\mathrm{O}$, Lee $\mathrm{SH}$, Jeong $\mathrm{SH}$, et al. A quantitative study of nanoparticle skin penetration with interactive segmentation. Med Biol Eng Comput. 2016;54(10):1469-1479.

14. Ryman-Rasmussen JP, Riviere JE, Monteiro-Riviere NA. Penetration of intact skin by quantum dots with diverse physicochemical properties. Toxicol Sci. 2006;91(1):159-165.

15. Labouta HI, El-Khordagui LK, Kraus T, Schneider M. Mechanism and determinants of nanoparticle penetration through human skin. Nanoscale. 2011;3(12):4989-4999.

16. Samberg ME, Oldenburg SJ, Monteiro-Riviere NA. Evaluation of silver nanoparticle toxicity in skin in vivo and keratinocytes in vitro. Environ Health Perspect. 2010;118(3):407-413.

17. Senzui M, Tamura T, Miura K, Ikarashi Y, Watanabe Y, Fujii M. Study on penetration of titanium dioxide ( $\mathrm{TiO} 2)$ nanoparticles into intact and damaged skin in vitro. $J$ Toxicol Sci. 2010;35(1):107-113.

18. Zvyagin AV, Zhao X, Gierden A, Sanchez W, Ross JA, Roberts MS. Imaging of zinc oxide nanoparticle penetration in human skin in vitro and in vivo. J Biomed Optics. 2008;13(6):064031.

19. Netzlaff F, Schaefer UF, Lehr CM, et al. Comparison of bovine udder skin with human and porcine skin in percutaneous permeation experiments. Altern Lab Anim. 2006;34(5):499-513.

20. Godin B, Touitou E. Transdermal skin delivery: predictions for humans from in vivo, ex vivo and animal models. Adv Drug Deliv Rev. 2007; 59(11):1152-1161.

21. Roberts MS, Roberts MJ, Robertson TA, et al. In vitro and in vivo imaging of xenobiotic transport in human skin and in the rat liver. J Biophotonics. 2008;1(6):478-493.

22. Zhu HJ, Jung EC, Hui XY, Maibach H. Proposed human stratum corneum water domain in chemical absorption. J Appl Toxicol. 2016; 36(8):991-996. 
23. Labouta HI, El-Khordagui LK, Schneider M. Could chemical enhancement of gold nanoparticle penetration be extrapolated from established approaches for drug permeation? Skin Pharmacol Physiol. 2012;25(4):208-218.

24. Kuo TR, Wu CL, Hsu CT, et al. Chemical enhancer induced changes in the mechanisms of transdermal delivery of zinc oxide nanoparticles. Biomaterials. 2009;30(16):3002-3008.

25. McCullough JL, Snyder DS, Weinstein GD, Friedland A, Stein B. Factors affecting human percutaneous penetration of methotrexate and its analogs in vitro. J Invest Dermatol. 1976;66(2):103-107.

26. Tominaga K, Tojo K. Effect of environmental temperature on transdermal drug penetration. Biol Pharm Bull. 2010;33(12):1983-1987.

27. Trabaris M, Laskin JD, Weisel CP. Effects of temperature, surfactants and skin location on the dermal penetration of haloacetonitriles and chloral hydrate. J Expo Sci Environ Epidemiol. 2012;22(4):393-397.

28. Lademann J, Richter H, Schaefer UF, et al. Hair follicles - a longterm reservoir for drug delivery. Skin Pharmacol Physiol. 2006;19(4): 232-236.

29. Lademann J, Richter H, Teichmann A, et al. Nanoparticles - an efficient carrier for drug delivery into the hair follicles. Eur J Pharm Biopharm. 2007;66(2):159-164.

30. Lademann J, Ilgevicius A, Zurbau O, et al. Penetration studies of topically applied substances: optical determination of the amount of stratum corneum removed by tape stripping. J Biomed Optics. 2006;11(5): 054026 .
31. Zhang LW, Monteiro-Riviere NA. Assessment of quantum dot penetration into intact, tape-stripped, abraded and flexed rat skin. Skin Pharmacol Physiol. 2008;21(3):166-180.

32. Zhang LW, Yu WW, Colvin VL, Monteiro-Riviere NA. Biological interactions of quantum dot nanoparticles in skin and in human epidermal keratinocytes. Toxicol Appl Pharmacol. 2008;228(2):200-211.

33. Gopee NV, Roberts DW, Webb P, et al. Quantitative determination of skin penetration of PEG-coated CdSe quantum dots in dermabraded but not intact SKH-1 hairless mouse skin. Toxicol Sci. 2009;111(1): 37-48.

34. Gratieri T, Schaefer UF, Jing LH, et al. Penetration of quantum dot particles through human skin. J Biomed Nanotechnol. 2010;6(5): 586-595.

35. Jeong SH, Kim JH, Yi SM, et al. Assessment of penetration of quantum dots through in vitro and in vivo human skin using the human skin equivalent model and the tape stripping method. Biochem Biophys Res Commun. 2010;394(3):612-615.

36. Prow TW, Monteiro-Riviere NA, Inman AO, et al. Quantum dot penetration into viable human skin. Nanotoxicology. 2012;6(2):173-185.
International Journal of Nanomedicine

\section{Publish your work in this journal}

The International Journal of Nanomedicine is an international, peerreviewed journal focusing on the application of nanotechnology in diagnostics, therapeutics, and drug delivery systems throughout the biomedical field. This journal is indexed on PubMed Central, MedLine, CAS, SciSearch $®$, Current Contents $\AA /$ Clinical Medicine,

\section{Dovepress}

Journal Citation Reports/Science Edition, EMBase, Scopus and the Elsevier Bibliographic databases. The manuscript management system is completely online and includes a very quick and fair peer-review system, which is all easy to use. Visit http://www.dovepress.com/ testimonials.php to read real quotes from published authors. 